\title{
Dental Imaging With Near-Infrared Transillumination Using Random Fiber Laser
}

\author{
Jiayu GUO, Yunjiang RAO, Weili ZHANG*, Zewen CUI, \\ Anran LIU, and Yongmei YAN
}

Key Laboratory of Optical Fiber Sensing \& Communications (Ministry of Education), University of Electronic Science and Technology of China, Chengdu 611731, China

*Corresponding author: Weili ZHANGＥ-mail: wl_zhang@uestc.edu.cn

\begin{abstract}
Contrary to the conventional detection method like radiography, the near infrared light source has been demonstrated to be suitable for dental imaging due to different reflectivity among enamel, dentin, and caries lesion. In this paper, three light sources with different bandwidths based on a transillumination method are compared. The contrast among enamel, dentin, and caries lesion is calculated in different situations. The experimental results show that the random fiber laser has the best comprehensive quality in dental imaging due to its high spectral density, low coherence, and deep penetration. This work provides a guidance for light source selection in dental imaging.
\end{abstract}

Keywords: Random fiber laser; near infrared; dental imaging; caries detection

Citation: Jiayu GUO, Yunjiang RAO, Weili ZHANG, Zewen CUI, Anran LIU, and Yongmei YAN, "Dental Imaging With Near-Infrared Transillumination Using Random Fiber Laser," Photonic Sensors, 2020, 10(4): 333-339.

\section{Introduction}

Dental caries is the most common oral disease, and the conventional dental detection methods are mainly visual inspection and radiography. Clearly visible caries can be detected by visual inspection and dental probe, while those hidden and inaccessible lesions mainly rely on dental radiography. However, these methods could underestimate or even miss the depth or size of the lesions if caries is at an early stage [1]. Near-infrared (NIR) transillumination (TI), a nonionizing imaging method with high sensitivity and easy operation has been investigated and demonstrated to be a promising technology to fill this gap [2-11].

The principle of TI is based on the difference in scattering and absorption of NIR light depending on the degree of demineralization. The dental caries caused mineral loss increases the scattering coefficient, thus appearing as the dark region in image [12]. The scattering coefficient of enamel in the visible is more than 20 times higher than that in NIR, which gives NIR a high contrast of demineralization. The investigation into NIR light for dental imaging has been concentrated on its wavelength. The wavelength from $400 \mathrm{~nm}$ to $2350 \mathrm{~nm}$ has been measured to compare the contrast of caries lesion and healthy enamel, and the result shows that wavelength beyond $1400 \mathrm{~nm}$ performs better [13]. Kenneth $\mathrm{H}$. Chan et al. had the similar conclusion, and the reduced light scattering coefficient beyond $1300 \mathrm{~nm}$ was the main reason for the higher lesion contrast at the longer NIR wavelengths [14]. Although the wavelength of the

Received: 11 November 2019 / Revised: 25 February 2020

(C) The Author(s) 2020. This article is published with open access at Springerlink.com

DOI: $10.1007 / \mathrm{s} 13320-020-0582-5$

Article type: Regular 
NIR light source has been discussed, the light sources used in these researches are mainly narrow linewidth light sources, which could lead to the speckle pattern suffered from strong interference [14], and the qualities (bandwidth and speckle contrast) of the light source still remain to be investigated.

Random laser (RL) has been demonstrated to be a promising light source for speckle-free imaging. Owing to stimulated lasing emission from multiple scattering feedbacks within the disordered medium [15-20], RL has the low coherence and high spectral density at the same time. Random fiber laser (RFL) is akin to the RL, being the quasi one-dimensional version of the RL, which possesses the same nonconventional laser characteristic, rather than by the fixed mirror or fiber Bragg grating. The feedback is provided by a scattering medium [21-25], apart from advantages in RL, RFL has the qualities of quasi one-dimension and high output power, flexible lasing wavelength, and robust structure. The RFL is demonstrated to be suitable for high power low-coherence full-field real-time speckle free imaging [26-29].

In this paper, the RFL is introduced to be the light source of dental imaging. An erbium-doped fiber based amplified spontaneous emission (ASE) and a narrow linewidth laser (NLL) are used to be compared with the RFL, and the wavelengths of three light sources are chosen to be around $1550 \mathrm{~nm}$, which accords with the premium wavelength for dental imaging. The experimental results show that the RFL light source has the best comprehensive abilities for dental imaging, and the teeth images are as clear as broadband light source with no speckle pattern and the contrast between lesion and enamel is higher than that of the broadband light source, meaning that the RFL possesses the high imaging quality and lesion contrast. This work gives an instruction to the light source selection for dental imaging.

\section{Experimental setup and results}

The experimental setup is shown in Fig. 1, in which three light sources including the RFL, ASE, and NLL are injected into a spool of step-index multimode fiber [MMF, core diameter is $105 \mu \mathrm{m}$, and the numerical aperture numerical aperture (NA) is 0.24 , NewPion Photonics] with a length of $30 \mathrm{~m}$ to reduce the coherence of the light sources. Lens 1 with a focal length of $6.2 \mathrm{~mm}$ after the MMF is used to collimate the output light. A pair of polarizers are placed before and after the teeth samples to eliminate the incident light and make sure that the camera can only receive photon from the teeth samples. The teeth samples are five dental sections with different thicknesses (two 3-mm-thickness sections with and without lesion, one 5-mm-thickness section, one 7-mm-thickness section, and one 12-mm-thickness section without lesion). Lens 2 with a focal length of $30 \mathrm{~mm}$ is placed after the polarizer to adjust the focal length of dental sections. An infrared camera (Xenics, Bobcat-640-GigE) is located behind Lens 2 to collect the detail of the teeth samples.

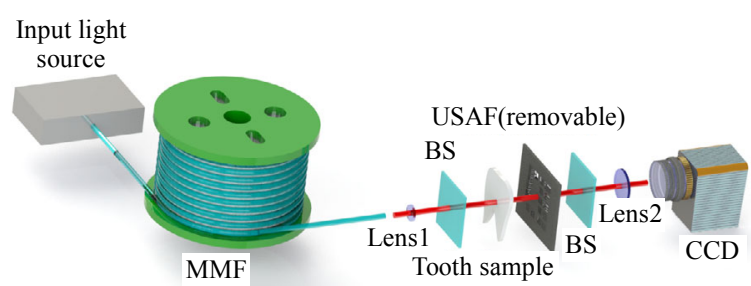

Fig. 1 Experimental setup. MMF: multimode fiber; USAF: US Air Force resolution chart; CCD: charge-coupled device camera.

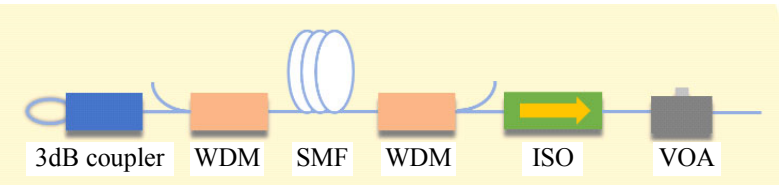

Fig. 2 Configuration of RFL. WDM: wavelength division multiplexer; SMF: single mode fiber; ISO: isolator; VOA: variable optical attenuator.

The configuration of the RFL is shown in Fig. 2. A $3 \mathrm{~dB}$ coupler and a 25-km-length SMF compose a half-opened structure where the RFL is generated through. Through a WDM, a $1455 \mathrm{~nm}$ Raman pump 
is injected into the SMF. After another WDM at the end of the SMF, the residual pump light is eliminated, and only the generated RFL is injected into the imaging part. The ISO can make sure that the laser is generated only through randomly distributed Rayleigh scattering.

The optical spectra of the RFL, ASE, and NLL are measured by an optical spectrum analyzer (OSA, Ando, spectral resolution of $0.01 \mathrm{~nm}$ ), as shown in Table 1 or referring to more details in our previous work in [22]. The $3 \mathrm{~dB}$ bandwidth of the RFL is $\sim 1.5 \mathrm{~nm}$, which is measured at the pump power of $33.4 \mathrm{dBm}$. The ASE has the $3 \mathrm{~dB}$ bandwidth of $\sim 7 \mathrm{~nm}$, with more than 4 times broader than that of the RFL. The $3 \mathrm{~dB}$ bandwidth of the NLL is less than $0.01 \mathrm{~nm}$. As we all know that the coherence length is inversely proportional to the central wavelength, the RFL has much shorter coherence length than NLL, and with the decoherence of a spool of the MMF, the coherence of the RFL is low enough to fulfill speckle free imaging. Our previous work has demonstrated that the multimode RFL can reach a speckle contrast of $\sim 0.049$, which is near the threshold of human perception and is enough for speckle-free imaging [23].

Table 1 Optics spectrum.

\begin{tabular}{cccc}
\hline Light source & RFL & ASE & NLL \\
\hline Central wavelength (nm) & 1556 & 1530 & 1549 \\
Bandwidth $(\mathrm{nm})$ & 1.5 & 7 & 0.01 \\
\hline
\end{tabular}

The dental images of four sound teeth samples by three light sources are shown in Fig. 3. The output powers of three light sources are controlled at $-1.5 \mathrm{dBm}$. From Fig. 3(i), the NLL image blurs the dental image because of the high coherence caused interference. The highly coherent NLL is not capable of dental imaging since the speckle pattern could be misrecognized as lesion. Contrary to the NLL, the RFL and ASE have clear images in four samples, as shown in Figs.3(a)-3(h). It is because that after the decoherence of the MMF, the speckle contrasts of the RFL and ASE decrease and weak interferences do not lead to a speckle pattern. Lesions in Samples 2 and 4 can be identified clearly in the RFL and ASE images, and enamel and dentin in four samples can be easily distinguished in the RFL and ASE images, hence, we can deduct from these figures that the RFL and ASE have a potential for dental imaging.

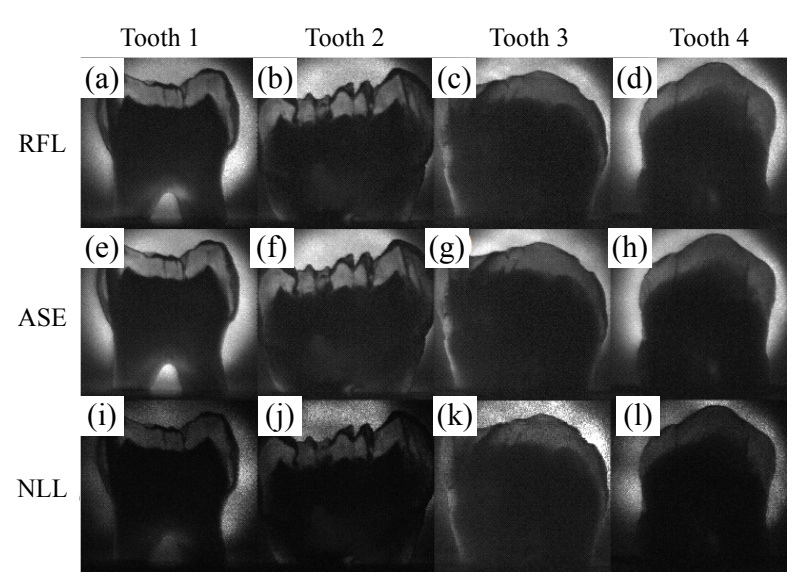

Fig. 3 Teeth image.

In order to give a quantitative comparison evaluation of the imaging quality, we choose to calculate the contrast between the enamel and background because in the trans-illumination configuration, the highly transmitted enamel can better express the quality of imaging. The enamel to noise contrast has been calculated for three light sources. The contrast is defined as

$$
C_{e}=\left(I_{e}-I_{b}\right) /\left[\left(\sigma_{e}+\sigma_{b}\right) / 2\right]
$$

where $C_{e}$ is the enamel to noise contrast, $I_{e}$ is the average intensity of enamel, and $I_{b}$ is the average intensity of the background. $\sigma_{e}$ and $\sigma_{b}$ refer to the standard deviation of the enamel and background, respectively. The contrast ratios of four teeth samples with three light sources are shown in Fig. 4. At the same light source, the contrast ratios of Teeth 1 and 2 are half bigger than those of Teeth 3 and 4 , it is because the thicknesses of Teeth 3 and 4 are bigger than those of Teeth 1 and 2, thus their intensities detected by the CCD are lower than those of Teeth 1 and 2, which causes the comparatively lower contrast ratio. The RFL and ASE share the 
similar contrast ratio in all 4 teeth samples and are significantly higher than that of the NLL. This result is in accord with our previous work about these three light sources [22]. Strong interference occurs in the high coherent NLL, which leads to speckle patterns that increase the standard deviation of enamel and reduce the contrast significantly. The broad band gives ASE the shortest coherent length, and with the decoherence of a spool of the MMF, the ASE has the lowest spatial coherence, which causes the highest contrast ratio. Benefitting from its multiple scattering feedback, the RFL can stimulate more spatial modes in the MMF, therefore reducing the speckle contrast remarkably. Since the RFL and ASE have comparable contrast ratios while the RFL is the result of stimulated lasing and possesses high spectral density, the RFL has the best comprehensive quality in enamel imaging.

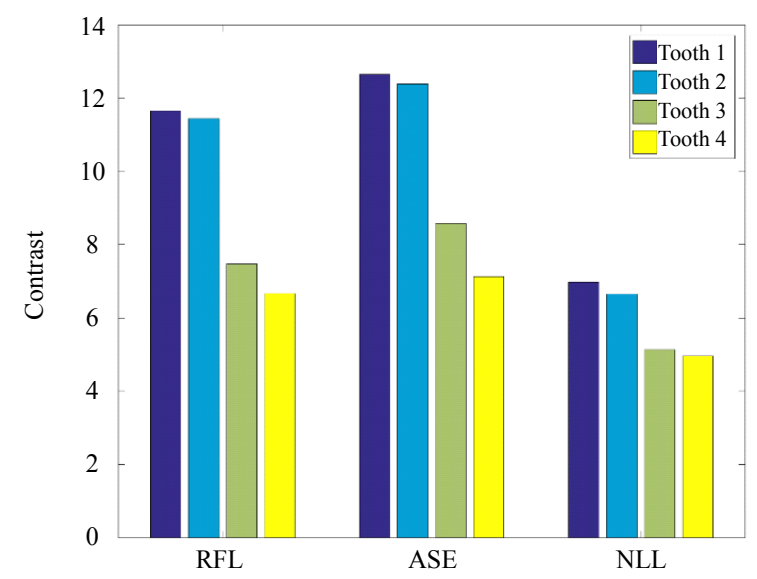

Fig. 4 Enamel to noise contrast.

Tooth with caries has also been measured to further compare the imaging quality among three light sources, as shown in Fig. 5. Similar to sound teeth samples in Fig. 3, the RFL and ASE images have better qualities than that of the NLL. Enamel and dentin can be identified clearly, so is caries lesion among red dotted line. However, it is hard to determine whether it is caries lesion or speckle pattern in the NLL image. The RFL and ASE outperform the NLL in caries lesion detection.

The intensity fluctuation near the caries region (along the red dotted line in Fig. 5) has also been investigated, as shown in Fig. 6. As mentioned before, the caries will cause a significant increase in the scattering coefficient, therefore in transillumination configuration, the caries region reflects more photons and has the lower intensity than that of sound enamel, in the pit in Fig. 6 referring to caries lesion. The NLL curve fluctuates a lot, which is caused by the interference, and the high deviation in cases above arises from this reason. The RFL and ASE have nearly the same intensity trend both in sound enamel and caries lesion, except that the RFL image has a little higher intensity in the enamel region. Caries to enamel ratio has been calculated to compare the quality of light sources, and the ratio is defined as $\left(I_{c}-I_{e}\right) /\left(I_{c}+I_{e}\right)$, where $I_{c}$ and $I_{e}$ refer to the average intensities of caries and enamel, respectively. Because of the interference caused intensity fluctuation, the NLL image cannot reflect intensity information precisely, and we do not take it into comparison. The ratios for the RFL and ASE are 0.74 and 0.72, the closer the ratio is to 1 , the higher the contrast is. The RFL and ASE have relatively high ratios, meaning that both two light sources have good performance in caries detection.

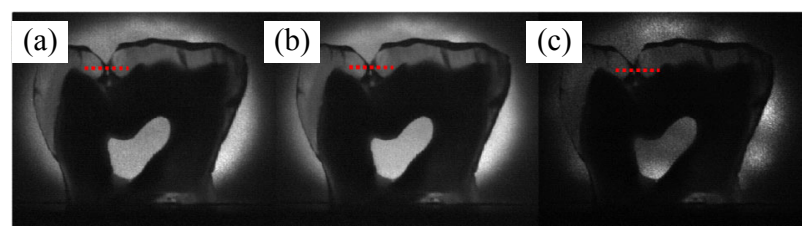

Fig. 5 Images of diseased tooth: (a) RFL image, (b) ASE image, and (c) NLL image.

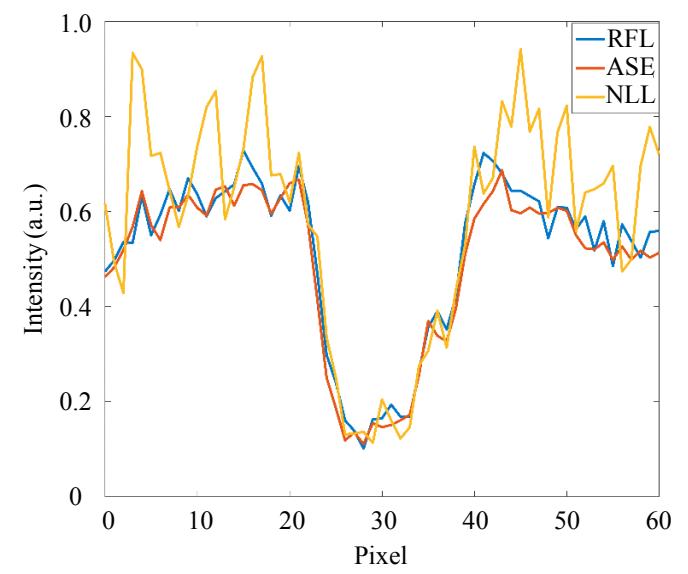

Fig. 6 Intensity fluctuation of the caries region. 


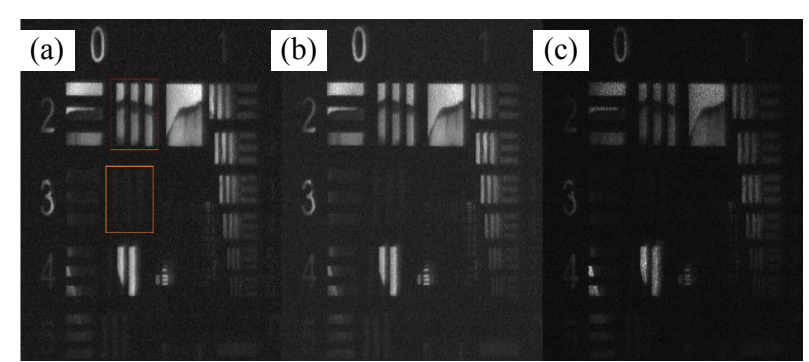

Fig. 7 USAF image.

A US Air Force (USAF) resolution chart has been placed behind the tooth sample for contrast calculation, as shown in Fig. 7. Two regions (the enamel region in the red rectangle and the dentin region in the orange rectangle) have been chosen to calculate the contrasts for enamel and dentin, respectively. The enamel to noise contrast is shown in (1), and the dentin to noise contrast is expressed as

$$
C_{d}=\left(I_{d}-I_{b}\right) /\left[\left(\sigma_{d}+\sigma_{b}\right) / 2\right]
$$

where $C_{d}$ is the dentin to noise contrast, $I_{e}$ and $I_{d}$ are the average intensities of enamel and dentin in transmission bar, and $I_{b}$ is the average intensity of the background, and $\sigma_{e}, \sigma_{d}$, and $\sigma_{b}$ refer to the standard deviations of enamel, dentin, and background, respectively. The contrast values are shown in Fig. 8. For the enamel to background ratio, the RFL and ASE have the similar value, which are about $40 \%$ higher than that of the NLL. The high coherence of the NLL raises the standard deviation of enamel and dentin, therefore decreasing the contrast. This result conforms with the $C_{e}$ in Fig.4 above. As for dentin to background ratio, the dentin reflects most of the photon because of its low transmittance, so the intensity of dentin is much lower than that of enamel, and the contrast for dentin is also much lower than that of enamel. In this case, because of its high spectral density and low coherence, the RFL has more photon penetrating from dentin, and the standard deviation of dentin is relatively low, thus having the highest contrast value, which means that the RFL has deeper penetration depth than that of the ASE. The picture illuminated by the ASE has lower contrast because the bandwidth of the ASE is more than 4 times broader than that of the RFL, and the low spectral density makes it harder for photons to penetrate from dentin (e.g., the ASE suffers from more loss compared with the RFL at the same operation power). The imaging performance of the RFL in highly transmissive enamel is comparable with the ASE, but in highly reflective dentin, the RFL is more preferable than the ASE.

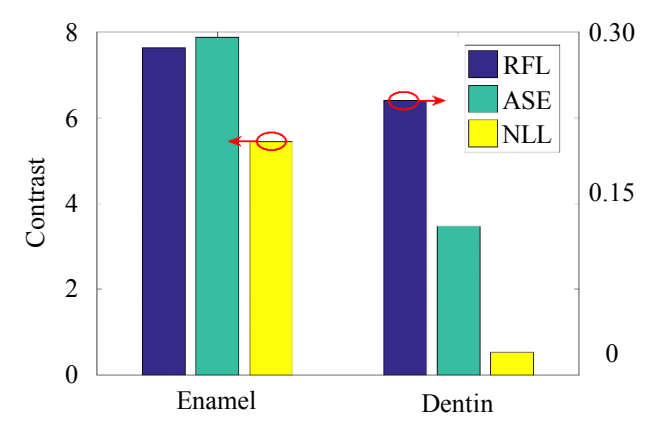

Fig. 8 Contrast ratios of enamel and dentin.

\section{Conclusions}

In conclusion, three light sources including the RFL, ASE, and NLL in the same wavelength region have been introduced to illuminate four sound teeth samples and a diseased tooth sample. The experimental results show that benefitting from its moderate bandwidth, the RFL has high spectral density and low coherence (having the clear image, high contrast, and deep penetration depth), which is more preferable than other light sources like the ASE and NLL for speckle free imaging at the limited operation power. The RFL is an ideally suitable light source for identifying enamel, dentin, and caries. A reference for light source selection in caries detection is provided in this paper.

\section{Acknowledgment}

This work was supported in part by the National Natural Science Foundation of China (Grant Nos. 11974071 and 61635005) and in part by Sichuan Science and Technology Program (Grant No. 2018HH0148). We thank Prof. Anderson Gomes in Universidade Federal of Pernambuco for providing 
the teeth samples and useful discussions.

Open Access This article is distributed under the terms of the Creative Commons Attribution 4.0 International License (http://creativecommons.org/ licenses/by/4.0/), which permits unrestricted use, distribution, and reproduction in any medium, provided you give appropriate credit to the original author(s) and the source, provide a link to the Creative Commons license, and indicate if changes were made.

\section{References}

[1] G. K. Stookey and C. Gonzalez-Cabezas, "Emerging methods of caries diagnosis," Journal of Dental Education, 2001, 65(10): 1001-1006.

[2] R. C. Lee, Y. X. Zhou, S. Finkleman, A. Sadr, and E. J. Seibel, "Near-infrared imaging of artificial enamel caries lesions with a scanning fiber endoscope," Sensors, 2019, 19(6): 1419.

[3] C. Ng, E. C. Almaz, J. C. Simon, D. Fried, and C. L. Darling, "Near-infrared imaging of demineralization on the occlusal surfaces of teeth without the interference of stains," Journal of Biomedical Optics, 2019, 24(3): 036002.

[4] F. Casalegno, T. Newton, R. Daher, M. Abdelaziz, A. Lodi-Rizzini, F. Schurmann, et al., "Caries detection with near-infrared transillumination using deep learning," Journal of Dental Research, 2019, 98(11): 1227-1233.

[5] W. A. Fried, D. Fried, K. H. Chan, and C. L. Darling, "High contrast reflectance imaging of simulated lesions on tooth occlusal surfaces at near-IR wavelengths," Lasers in Surgery and Medicine, 2013, 45(8): 533-541.

[6] J. Gomez, "Detection and diagnosis of the early caries lesion," BMC Oral Health, 2015, 15(1): S3.

[7] S. A. Son, K. H. Jung, C. C. Ko, and Y. H. Kwon, "Spectral characteristics of caries-related autofluorescence spectra and their use for diagnosis of caries stage," Journal of Biomedical Optics, 2016, 21(1): 015001.

[8] M. A. Geibel, S. Carstens, U. Braisch, A. Rahman, M. Herz, and A. Jablonski-Momeni, "Radiographic diagnosis of proximal caries-influence of experience and gender of the dental staff," Clinical Oral Investigations, 2017, 21(9): 2761-2770.

[9] R. W. Evans, C. A. Feldens, and P. Phantunvanit, "A protocol for early childhood caries diagnosis and risk assessment," Community Dentistry and Oral Epidemiology, 2018, 46(5): 518-525.

[10] J. K. Mitchell, A. R. Furness, R. J. Sword, S. W. Looney, W. W. Brackett, and M. G. Brackett, "Diagnosis of pit-and-fissure caries using three-dimensional scanned images," Operative
Dentistry, 2018, 43(3): E152-E157.

[11] J. C. Simon, S. A. Lucas, M. Staninec, H. Tom, K. H. Chan, C. L. Darling, et al., "Near-IR transillumination and reflectance imaging at 1,300 $\mathrm{nm}$ and 1,500-1,700 $\mathrm{nm}$ for in vivo caries detection," Lasers in Surgery and Medicine, 2016, 48(9): 828-836.

[12] L. Karlsson, A. M. A. Maia, B. B. C. Kyotoku, S. Tranaeus, A. S. L. Gomes, and W. Margulis, "Near-infrared transillumination of teeth: measurement of a system performance," Journal of Biomedical Optics, 2010, 15(3): 036001.

[13] V. B. Yang, D. A. Curtis, and D. Fried, "Cross-polarization reflectance imaging of root caries and dental calculus on extracted teeth at wavelengths from 400 to $2350 \mathrm{~nm}$," Journal of Biophotonics, 2018, 11(11): e201800113.

[14] K. H. Chan and D. Fried, "Multispectral cross-polarization reflectance measurements suggest high contrast of demineralization on tooth surfaces at wavelengths beyond $1300 \mathrm{~nm}$ due to reduced light scattering in sound enamel," Journal of Biomedical Optics, 2018, 23(6): 060501.

[15] N. M. Lawandy, R. M. Balachandran, A. S. L. Gomes, and E. Sauvain, "Laser action in strongly scattering media," Nature, 1994, 368(6470): 436-438.

[16] H. Cao, Y. G. Zhao, S. T. Ho, E. W. Seelig, Q. H. Wang, and R. P. H. Chang, "Random laser action in semiconductor powder," Physical Review Letters, 1999, 82(11): 2278-2281.

[17] D. S. Wiersma, "The physics and applications of random lasers," Nature Physics, 2008, 4(5): 359-367.

[18] M. Leonetti, C. Conti, and C. Lopez, "The mode-locking transition of random lasers," Nature Photonics, 2011, 5(10): 615-617.

[19] B. Redding, M. A. Choma, and H. Cao, "Speckle-free laser imaging using random laser illumination," Nature Photonics, 2012, 6(6): 355-359.

[20] S. Schonhuber, M. Brandstetter, T. Hisch, C. Deutsch, M. Krall, H. Detz, et al., "Random lasers for broadband directional emission," Optica, 2016, 3(10): 1035-1038.

[21] S. K. Turitsyn, S. A. Babin, A. E. El-Taher, P. Harper, D. V. Churkin, S. I. Kablukov, et al., "Random distributed feedback fibre laser," Nature Photonics, 2010, 4(4): 231-235.

[22] R. Ma, Y. J. Rao, W. L. Zhang, and B. Hu, "Multimode random fiber laser for speckle-free imaging," IEEE Journal of Selected Topics in Quantum Electronics, 2019, 25(1): 1-6.

[23] D. V. Churkin, S. Sugavanam, I. D. Vatnik, Z. Wang, E. V. Podivilov, S. A. Babin, et al., "Recent advances in fundamentals and applications of random fiber lasers," Advances in Optics and Photonics, 2015, 
7(3): 516-569.

[24] R. Ma, W. L. Zhang, J. Y. Guo, and Y. J. Rao, "Decoherence of fiber supercontinuum light source for speckle-free imaging," Optics Express, 2018, 26(20): 26758-26765.

[25] R. Ma, J. Q. Li, J. Y. Guo, H. Wu, H. H. Zhang, B. $\mathrm{Hu}$, et al., "High-power low spatial coherence random fiber laser," Optics Express, 2019, 27(6): 8738-8744.

[26] B. H. Hokr, M. S. Schmidt, J. N. Bixler, P. N. Dyer, G. D. Noojin, B. Redding, et al., "A narrow-band speckle-free light source via random Raman lasing," Journal of Modern Optics, 2016, 63(1): 46-49.

[27] B. H. Hokr, D. T. Nodurft, J. V. Thompson, J. N.
Bixler, G. D. Noojin, B. Redding, et al., "Lighting up microscopy with random Raman lasing," Real-Time Measurements, Rogue Events, and Emerging Applications, San Francisco, CA, 2016, pp: 9732.

[28] M. T. Carvalho, A. S. Lotay, F. M. Kenny, J. M. Girkin, and A. S. L. Gomes, "Random laser illumination: an ideal source for biomedical polarization imaging?" Multimodal Biomedical Imaging XI, San Francisco, CA, 2016, pp: 9701.

[29] B. Redding, P. Ahmadi, V. Mokan, M. Seifert, M. A. Choma, and H. Cao, "Low-spatial-coherence high-radiance broadband fiber source for speckle free imaging," Optics Letters, 2015, 40(20): $4607-4610$. 